\title{
Regard sur l'évolution de l'éducation permanente dans les universités du Québec The Evolution of Continuing Education in Quebec Universities
}

\author{
MAURICE BARBEAU*
}

Si les sciences de la vie ne nous avaient familiarisés avec les conditions de l'évolution des espèces nous pourrions tout aussi bien nous étonner des résistances que des prosélytismes à l'endroit de l'éducation permanente, phénomène qui, pour prendre toute sa signification, doit être saisi dans la foulée du mouvement pédagogique des cinquante dernières années. Jamais n'a-t-on autant parlé d'éducation alors que jamais l'éducation, comme intervention humaine finalisée, surtout auprès de jeunes, n'a connu autant de résistance de la part des forces de l'environnement - dont l'action reste par contre toujours sporadique. Quiconque aspire à des transformations radicales et immédiates aurait profit à se rappeler que, comme dit Alain, "Porter le Monde, comme Atlas, cela vous fait des épaules; mais le bon sens n'y gagne rien. Porter n'est pas comprendre".

C'est d'ailleurs dans une perspective de cheminement graduel qu'il est intéressant d'examiner le mouvement de l'institution-nalisation de l'éducation permanente au Québec, la dimension québécoise n'étant bien sûr qu'une facette d'un phénomène universel. Sans nous arrêter à des discussions sémantiques, le phénomène éducation permanente peut s'analyser comme la résultante de trois forces conjuguées: un appétit de culture des individus avivé par un environnement toujours plus enrichi, une valorisation des éléments de culture, partant de l'expérience personnelle, par la puissance des moyens de communication, ainsi que la nécessité de satisfaire aux besoins de plus en plus exigents des masses sous-développées, au fond autant d'éléments qu'intègre un nouvel humanisme.

Les universités anglophones du Québec ont fait le pont depuis longtemps par leurs services d'extension qui continuent ainsi une tradition nord-américaine de plus d'un siècle généralement bien connue. Les universités francophones, par contre, pour des raisons qu'il serait trop long d'analyser, affrontent plus intensément un choc en retour. Voici donc quelques faits majeurs laissant percevoir où en sont les universités francophones dans leur démarche vers une intégration progressive des éléments d'éducation permanente. L'ordre de présentation traduit sa signification.

Comme la plupart des universités, Laval a une assez longue tradition d'éducation permanente sous forme de promotion culturelle: cours d'été pour les enseignants dès les années ' 40 , B.A. pour adultes ('50), programme sur la coopération . . . Dès ' 62 , lors de sa 
création, l'Extension de l'enseignement universitaire devient responsable "de toutes les catégories d'étudiants qui n'entrent pas dans la définition d'étudiants réguliers". En '67, le Conseil de l'Université instituait une "Direction générale de l'éducation des adultes" dont lé rôle a été principalement de faciliter l'admission dans les facultés, au titre d'étudiants réguliers, des adultes qui ne répondaient pas aux conditions régulières d'admission, et cela par la voie d'une scolarité d'appoint requise du candidat après négociation avec les facultés. L'apport de Laval sous cet aspect constituait un certain précédent dans la perspective de "l'université de la seconde chance".

Dès '72, la Commission de la réforme recommandait de "passer de l'enseignement et de l'éducation des adultes à l'éducation permanente conçue comme une philosophie". D'où la phase d'intégration, sous le Service de l'éducation permanente, des directions de la "Direction de l'éducation permanente", de l'Extension, des Cours d'été, de la Formation des maîtres et du B.A. pour adultes. Et finalement, en '75, la mise en place, selon la recommandation du Conseil des universités, du "Service de promotion de l'éducation permanente". C'était la consécration de l'éducation permanente "comme principe de l'ensemble" de l'établissement. Ce service aura dorénavant la tâche d'animer l'institution dans le sens d'une trajectoire orientée selon les conditions de l'éducation permanente, d'inventorier les besoins des clientèles, d'inciter les facultés à y répondre par, entre autres moyens, des cours d'été, du soir et hors campus. Avec, comme corollaire nécessaire, la création et l'organisation, sous l'autorité du secrétaire général, d'un Service d'accueil dont l'assistance est spécifiquement limitée aux adultes ne répondant pas aux conditions régulières d'admission. Tandis que l'Extension continue à administrer des programmes spéciaux et à offrir des cours compensateurs requis par le cheminement des adultes. Ainsi donc, en matière d'éducation permanente, Laval consacre le principe d'une intégration institutionnelle et crée un organisme d'accueil des adultes double mesure qui confère à l'institution une certaine originalité.

Après une assez longue expérience dans le champ de l'éducation des adultes: B.A. pour adultes à temps partiel '52 et son Service d'éducation permanente '68, l'Université de Montréal adoptait la Faculté d'éducation permanente comme mode d'organisation. Cette structure garantissait une plus grande autonomie administrative, éliminait bien des partenaires et augmentait le pouvoir décisionnel de la faculté face à un volume d'activités très considérable. L'orientation et la gouverne de la Faculté d'éducation permanente sont assurées par un Comité de l'éducation permanente rattaché à'l'Assemblée universitaire et un Comité de l'éducation continue rattaché à la Commission des études. La direction immédiate est assumée par le doyen, assisté de trois vice-doyens et d'un Conseil de faculté.

Ce mode d'organisation en faculté est unique dans les universités du Québec et paraît une solution pratique inspirée avant tout des exigences administratives d'une clientèle d'environ 11000 étudiants. Nous sommes visiblement devant une solution de compromis qui n'est ni l'intégration totale des activités d'éducation permanente à l'ensemble de l'Université ni une entité parallèle supposant un champ d'enseignement relativement bien défini comme c'est le cas de la plupart des autres facultés. La Faculté d'éducation permanente n'a de juridiction qu'au niveau des études de premier cycle et que sur des programmes s'adressant à la clientèle adulte.

De surcroît il est intéressant de noter que la Faculté d'éducation permanente de l'Université de Montréal consacre d'avantage ses efforts à répondre aux besoins des professions 
nouvelles (animation ... ) ou en transformation (enseignants ... ). Pour y parvenir elle a créé des postes de responsables de programmes dont le rôle est d'assurer, après une analyse, en collaboration avec les intéressés, de leurs besoins et des façons d'y satisfaire, la mise en marche et le contrôle des programmes qui en émanent. La fonction de responsable de programme prend alors une signification très engagée, enrichissante à la fois pour ceux qui en sont titulaires, pour l'institution et pour les groupes desservis. Le service à la collectivité comme fonction de l'Université prend ainsi une nouvelle dimension.

A L'Université de Sherbrooke, l'Extension de l'enseignement s'est transformée subséquemment en "Service des cours à temps partiel "qui est devenu, en '72, la Direction générale de l'éducation permanente, celle-ci se définissant comme un organisme de "type exécutif", dont le rôle est "d'assurer, en collaboration avec les facultés et services de l'Université", le développement et le fonctionnement des projets adoptés pour fins d'éducation des adultes et d'éducation permanente". En outre, il est dévolu à la Direction générale de l'éducation permanente "d'assurer le travail d'animation et de coordination nécessaire à la réalisation de projets spécifiques d'éducation des adultes et d'éducation permanente". A y regarder de près, à supposer que les dénominations veuillent vraiment traduire des nuances, nous serions en présence d'un premier niveau d'intégration, puisque la Direction générale de l'éducation permanente se consacre à l'administration des programmes des facultés au bénéfice des adultes à temps partiel. Il importe également de faire mention qu'un organisme, désigné sous l'appellation "Fer de lance" et qui en est un de concertation régionale, regroupe, sous l'égide de l'Université de Sherbrooke, les services d'éducation des adultes du CEGEP de Sherbrooke et des régionales de l'Estrie et d'Eastern Township à des fins de planification et de coopération. Pour la première fois, les échanges milieu - université donnent naissance à un mécanisme d'approche et d'action concertée.

L'Université du Québec, avec son réseau de dix unités conștituantes, couvre un spectre de relations étendues pour répondre aux besoins de ses quelque 30000 étudiants dont plus de $60 \%$ sont à temps partiel, et dont bon nombre sont des adultes.

Le Service de l'éducation permanente de l'UQAM offre une programmation en vue de l'enseignement des langues pour satisfaire à la clientèle de son "Ecole française", pourvoit au perfectionnement des enseignants et dispense un certain nombre de cours dans une perspective de promotion culturelle demeurant ainsi dans le sens de la tradition universitaire. Par contre, là ou l'innovation parait certaine c'est par l'action de sa section "Développement et service à la collectivité" auprès des milieux défavorisés. Le protocole signé avec la CSN et la FTQ révèle l'ampleur et l'intensité de son intervention dans son milieu d'influence.

Devant l'accroissement considérable de ses étudiants à temps partiel la constituante des Trois-Rivières abolissait, en février '76, son "Service de l'éducation permanente et de l'animation pédagogique" pour le remplacer par un "Service de développement pédagogique" dont l'action, au plan éducation permanente, se perçoit du fait que les responsabilités de cet organisme couvrent "la promotion des programmes destinés aux étudiants en situation particulière et la coordination des programmes de coopération avec l'extérieur".

C'est peut-être à l'UQAR qu'il faut chercher l'une des dimensions les plus originales et les plus significatives d'un point de vue prospectif. A Rimouski, c'est le "Secteur des études à temps partiel" qui est responsable des adultes. Un doyen adjoint, rattaché au doyen des études de premier cycle, pourvoit aux besoins des étudiants adultes à temps partiel, en collaboration avec un doyen adjoint qui s'occupe des étudiants réguliers. L'un et l'autre voient 
“à ce que les cours dispensés aux étudiants réguliers satisfassent aux objectifs d'un programme donné et aux attentes des étudiants".

Des quelque 3300 étudiants admis à l'Université du Québec à Rimouski, 2500 le sont à temps partiel. De toute évidence c'est-là une situation qui reflète les besoins de la clientèle, besoins qui sont eux-mêmes fonction de conditions géographiques particulières. La région desservie par l'UQUAR recouvre à peu près tout l'est de la province depuis la Rivière-duLoup. De là la double exigence de la priorité donnée aux études à temps partiel pour répondre plus spécialement aux besoins des enseignants, ainsi que, par suite d'une clientèle relativement restreinte, de la nécessité d'une intégration la plus poussée possible des programmes pour adultes aux études régulières. De fait, l'éducation des adultes devient la raison d'être de l'UQUAR.

Nous assistons alors à une décentralisation très poussée des services d'enseignement. La dispersion de la clientèle depuis des endroits comme Tadoussac, les Iles de la Madeleine, Gaspé, sur un territoire très vaste et aux communications difficiles, rend aussi l'encadrement difficile. Le territoire est alors divisé en septs régions administratives dans lesquelles l'UQUAR maintient des bureaux régionaux confiés à des agents de liaison dont la tâche en est principalement une d'animation, d'étude des besoins et d'organisation de l'enseignement aux adultes. On perçoit comment une telle décentralisation administrative coute cher et rend l'encadrement embarrassant.

La clientèle serévèle bien sûr hétérogène et très dispersée. Et pour répondre à ces besoins, il va de soi que, en conséquence, la communication pédagogique doit se plier à des contraintes imposées par des groupes nécessairement restreints alors que, par la force des choses, les professeurs deviennent itinérants.

Un coup d'oeil du côté des université francophones hors du Québec révèle par exemple que l'université d'Ottawa, qu i a déjà une longue tradition d'éducation des adultes, vient récemment d'accepter l'accès aux études à temps partiel à tous les niveaux d'enseignement: baccalauréat, maitrise et doctorat. Cette mesure est complétée par des horaires d'enseignement depuis 8 h30 du matin jusqu'à 22 heures du soir, la rotation accrue des cours à option et la reconnaissance des cours d'été, du soir, hors-campus et par correspondance comme constituants de la charge normale des professeurs réguliers. L'Université St-Paul pour sa part, compte tenu de son orientation et des disciplines qu'elle dispense, dessert une clientèle d'adultes à temps partiel dans une proportion d'environ 75\%. A l'Université de Moncton, "tout cours ou activité d'apprentissage organisé à l'intention d'autres publics que les étudiants réguliers à temps complet doit être organisé par l'Education permanente ou avec son assentiment". Et pour des raisons analogues à celles que l'on retrouve à Rimouski, trois directeurs associés sont responsables des territoires de Bathurst-Shippegan, Moncton et Edmundston.

La tentation est facile de vouloir réunir en faisceau les diverses dominantes des mesures prises par les universités en matière d'éducation des adultes et d'éducation permanente et d'y voir les composantes essentielles d'un modèle de fonctionnement des universités. La réalité obéit à la logique des évènements qui est d'un autre ordre. Dans les faits, toutefois, l'évolution des universités, sous la dépendance de l'éducation permanente "comme principe d'intégration" se confirme progressivement. L'éducation des adultes sort de plus en plus de la marginalité. Et l'option entre l'intégration (Laval) et un organisme à pouvoirs spécifiques (Montréal) relève plutôt de conditions temporaires. La spécificité d'un traitement 
particulier répondant aux besoins des adultes est partout reconnue et devient un élément important des mesures adoptées par les universités. L'insertion progressive dans le milieu se révèle un facteur nettement déterminant sous l'impulsion de philosophies dont les nuances sont déjà perceptibles. Aussi, des mesures reliées à l'admission et aux horaires journaliers sont-elles également en place en bien des endroits. Il devient une fois de plus évident que, comme le dit Alain "Toute sagesse doit plus d'une couronne votive à la nécessité." 